\title{
Regular Expression Quantifiers - at least $m$ Occurrences
}

\author{
Michał Trybulec \\ Motorola Software Group \\ Cracow, Poland
}

\begin{abstract}
Summary. This is the second article on regular expression quantifiers. [4] introduced the quantifiers $m$ to $n$ occurrences and optional occurrence. In the sequel, the quantifiers: at least $m$ occurrences and positive closure (at least 1 occurrence) are introduced. Notation and terminology were taken from [8], several properties of regular expressions from [7].
\end{abstract}

MML identifier: FLANG_3 , version: $\underline{7.8 .05 \quad 4.89 .993}$

The notation and terminology used here are introduced in the following papers: [5], [1], [6], [2], [3], and [4].

\section{Preliminaries}

For simplicity, we follow the rules: $E, x$ denote sets, $A, B, C$ denote subsets of $E^{\omega}, a, b$ denote elements of $E^{\omega}$, and $k, l, m, n$ denote natural numbers.

The following proposition is true

(1) If $B \subseteq A^{*}$, then $\left(A^{*}\right)^{\frown} B \subseteq A^{*}$ and $B \frown A^{*} \subseteq A^{*}$.

\section{At LEAST $m$ OCCURRENCES}

Let us consider $E, A, n$. The functor $A^{n, . .}$ yielding a subset of $E^{\omega}$ is defined as follows:

(Def. 1) $\quad A^{n, . .}=\bigcup\left\{B: \bigvee_{m}\left(n \leq m \wedge B=A^{m}\right)\right\}$

We now state a number of propositions: 
(2) $x \in A^{n, \ldots}$ iff there exists $m$ such that $n \leq m$ and $x \in A^{m}$.

(3) If $n \leq m$, then $A^{m} \subseteq A^{n, . .}$.

(4) $A^{n, \ldots}=\emptyset$ iff $n>0$ and $A=\emptyset$.

(5) If $m \leq n$, then $A^{n, . \cdot} \subseteq A^{m, \cdot .}$.

(6) If $k \leq m$, then $A^{m, n} \subseteq A^{k, \cdot .}$.

(7) If $m \leq n+1$, then $A^{m, n} \cup\left(A^{(n+1), . \cdot}\right)=A^{m, \cdot .}$.

(8) $A^{n} \cup\left(A^{(n+1), . .}\right)=A^{n, . .}$.

(9) $A^{n, . .} \subseteq A^{*}$.

(10) \langle\rangle$_{E} \in A^{n, . .}$ iff $n=0$ or \langle\rangle$_{E} \in A$.

(11) $A^{n, . .}=A^{*}$ iff \langle\rangle$_{E} \in A$ or $n=0$.

(12) $A^{*}=A^{0, n} \cup\left(A^{(n+1), . .}\right)$.

(13) If $A \subseteq B$, then $A^{n, .} \subseteq B^{n, .}$.

(14) If $x \in A$ and $x \neq\langle\rangle_{E}$, then $A^{n, . .} \neq\left\{\langle\rangle_{E}\right\}$.

(15) $A^{n, \cdots}=\left\{\langle\rangle_{E}\right\}$ iff $A=\left\{\langle\rangle_{E}\right\}$ or $n=0$ and $A=\emptyset$.

(16) $\quad A^{(n+1), . .}=\left(A^{n, . .}\right) \frown A$.

(17) $\left(A^{m, . \cdot}\right) \frown A^{*}=A^{m, \cdot .}$.

(18) $\left(A^{m, . .}\right) \frown\left(A^{n, . .}\right)=A^{(m+n), . .}$.

(19) If $n>0$, then $\left(A^{m, \cdot .}\right)^{n}=A^{m \cdot n, \cdot .}$.

(20) $\left(A^{n, . .}\right)^{*}=\left(A^{n, . .}\right)$ ?.

(21) If $A \subseteq C^{m, . .}$ and $B \subseteq C^{n, . .}$, then $A \frown B \subseteq C^{(m+n), . .}$.

(22) $\quad A^{(n+k), . .}=\left(A^{n, . .}\right) \frown A^{k}$.

(23) $\quad A \frown\left(A^{n, . .}\right)=\left(A^{n, . .}\right) \frown A$.

(24) $\left(A^{k}\right) \frown\left(A^{n, . .}\right)=\left(A^{n, . .}\right) \frown A^{k}$.

(25) $\left(A^{k, l}\right) \frown\left(A^{n, . .}\right)=\left(A^{n, . .}\right) \frown A^{k, l}$.

(26) If \langle\rangle$_{E} \in B$, then $A \subseteq A \frown\left(B^{n, . .}\right)$ and $A \subseteq\left(B^{n, . .}\right) \frown A$.

(27) $\left(A^{m, . .}\right) \frown\left(A^{n, . .}\right)=\left(A^{n, . .}\right) \frown\left(A^{m, . .}\right)$.

(28) If $A \subseteq B^{k, . .}$ and $n>0$, then $A^{n} \subseteq B^{k, . .}$.

(29) If $A \subseteq B^{k, . .}$ and $n>0$, then $A^{n, . .} \subseteq B^{k, . .}$.

(30) $\left(A^{*}\right) \frown A=A^{1, . .}$.

(31) $\left(A^{*}\right) \frown A^{k}=A^{k, \cdot}$.

(32) $\left(A^{m, . \cdot}\right) \frown A^{*}=\left(A^{*}\right) \frown\left(A^{m, . \cdot}\right)$.

(33) If $k \leq l$, then $\left(A^{n, . .}\right) \frown A^{k, l}=A^{(n+k), . .}$.

(34) If $k \leq l$, then $\left(A^{*}\right) \frown A^{k, l}=A^{k, . .}$.

(35) $A^{m n, . .} \subseteq A^{m \cdot n, . .}$.

(36) $A^{m n, . .} \subseteq\left(A^{n, . .}\right)^{m}$.

(37) If $a \in C^{m, . .}$ and $b \in C^{n, . .}$, then $a^{\frown} b \in C^{(m+n), . .}$.

(38) If $A^{k, . .}=\{x\}$, then $x=\langle\rangle_{E}$. 
(39) If $A \subseteq B^{*}$, then $A^{n, . .} \subseteq B^{*}$.

(40) $A ? \subseteq A^{k, . .}$ iff $k=0$ or \langle\rangle$_{E} \in A$.

(41) $\left(A^{k, . .}\right) \frown A ?=A^{k, . .}$.

(42) $\left(A^{k, . \cdot}\right) \frown A ?=A ? \frown\left(A^{k, \cdot \cdot}\right)$.

(43) If $B \subseteq A^{*}$, then $\left(A^{k, .}\right) \frown B \subseteq A^{k, . .}$ and $B \frown\left(A^{k, .}\right) \subseteq A^{k, . .}$.

(44) $A \cap B^{k, . .} \subseteq\left(A^{k, . .}\right) \cap\left(B^{k, . .}\right)$.

(45) $\left(A^{k, . .}\right) \cup\left(B^{k, . .}\right) \subseteq(A \cup B)^{k, . .}$.

(46) $\langle x\rangle \in A^{k, . .}$ iff $\langle x\rangle \in A$ but \langle\rangle$_{E} \in A$ or $k \leq 1$.

(47) If $A \subseteq B^{k, . .}$, then $B^{k, . .}=(B \cup A)^{k, . .}$.

\section{Positive Closure}

Let us consider $E, A$. The functor $A^{+}$yielding a subset of $E^{\omega}$ is defined as follows:

(Def. 2) $A^{+}=\bigcup\left\{B: \bigvee_{n}\left(n>0 \wedge B=A^{n}\right)\right\}$.

Next we state a number of propositions:

(48) $x \in A^{+}$iff there exists $n$ such that $n>0$ and $x \in A^{n}$.

(49) If $n>0$, then $A^{n} \subseteq A^{+}$.

(50) $A^{+}=A^{1, . .}$.

(51) $A^{+}=\emptyset$ iff $A=\emptyset$.

(52) $A^{+}=\left(A^{*}\right) \frown A$.

(53) $\quad A^{*}=\left\{\langle\rangle_{E}\right\} \cup A^{+}$.

(54) $A^{+}=A^{1, n} \cup\left(A^{(n+1), . .}\right)$.

(55) $A^{+} \subseteq A^{*}$.

(56) \langle\rangle$_{E} \in A^{+}$iff \langle\rangle$_{E} \in A$.

(57) $A^{+}=A^{*}$ iff \langle\rangle$_{E} \in A$.

(58) If $A \subseteq B$, then $A^{+} \subseteq B^{+}$.

(59) $A \subseteq A^{+}$.

(60) $A^{*+}=A^{*}$ and $A^{+*}=A^{*}$.

(61) If $A \subseteq B^{*}$, then $A^{+} \subseteq B^{*}$.

(62) $A^{+^{+}}=A^{+}$.

(63) If $x \in A$ and $x \neq\langle\rangle_{E}$, then $A^{+} \neq\left\{\langle\rangle_{E}\right\}$.

(64) $A^{+}=\left\{\langle\rangle_{E}\right\}$ iff $A=\left\{\langle\rangle_{E}\right\}$.

(65) $A^{+} ?=A^{*}$ and $A ?^{+}=A^{*}$.

(66) If $a, b \in C^{+}$, then $a^{\frown} b \in C^{+}$.

(67) If $A \subseteq C^{+}$and $B \subseteq C^{+}$, then $A \frown B \subseteq C^{+}$.

(68) $A \frown A \subseteq A^{+}$. 
(69) If $A^{+}=\{x\}$, then $x=\langle\rangle_{E}$.

(70) $A^{\frown} A^{+}=A^{+\frown A}$.

(71) $\left(A^{k}\right)^{\frown} A^{+}=A^{+} \frown A^{k}$.

(72) $\left(A^{m, n}\right) \frown A^{+}=A^{+} \frown A^{m, n}$.

(73) If \langle\rangle$_{E} \in B$, then $A \subseteq A \frown B^{+}$and $A \subseteq B^{+} \frown A$.

(74) $A^{+} \frown A^{+}=A^{2, . .}$.

(75) $A^{+\frown} A^{k}=A^{(k+1), . .}$.

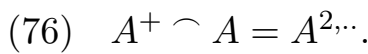

(77) $\quad$ If $k \leq l$, then $A^{+} \frown A^{k, l}=A^{(k+1), . .}$.

(78) If $A \subseteq B^{+}$and $n>0$, then $A^{n} \subseteq B^{+}$.

(79) $A^{+} \frown A ?=A ? \frown A^{+}$.

(80) $A^{+} \frown A ?=A^{+}$.

(81) $A ? \subseteq A^{+}$iff \langle\rangle$_{E} \in A$.

(82) If $A \subseteq B^{+}$, then $A^{+} \subseteq B^{+}$.

(83) If $A \subseteq B^{+}$, then $B^{+}=(B \cup A)^{+}$.

(84) If $n>0$, then $A^{n, . .} \subseteq A^{+}$.

(85) If $m>0$, then $A^{m, n} \subseteq A^{+}$.

(86) $\left(A^{*}\right) \frown A^{+}=A^{+} \frown A^{*}$.

(87) $A^{+k} \subseteq A^{k, . .}$.

(88) $A^{+m, n} \subseteq A^{m, . .}$.

(89) If $A \subseteq B^{+}$and $n>0$, then $A^{n, . \cdot} \subseteq B^{+}$.

(90) $A^{+\frown}\left(A^{k, . .}\right)=A^{(k+1), . .}$.

(91) $\quad A^{+\frown}\left(A^{k, . .}\right)=\left(A^{k, . .}\right) \frown A^{+}$.

(92) $A^{+} \frown A^{*}=A^{+}$.

(93) If $B \subseteq A^{*}$, then $A^{+} \frown B \subseteq A^{+}$and $B \frown A^{+} \subseteq A^{+}$.

(94) $(A \cap B)^{+} \subseteq A^{+} \cap B^{+}$.

(95) $A^{+} \cup B^{+} \subseteq(A \cup B)^{+}$.

(96) $\langle x\rangle \in A^{+}$iff $\langle x\rangle \in A$.

\section{REFERENCES}

[1] Grzegorz Bancerek. The fundamental properties of natural numbers. Formalized Mathematics, 1(1):41-46, 1990.

[2] Karol Pạk. The Catalan numbers. Part II. Formalized Mathematics, 14(4):153-159, 2006.

[3] Michał Trybulec. Formal languages - concatenation and closure. Formalized Mathematics, 15(1):11-15, 2007.

[4] Michał Trybulec. Regular expression quantifiers $-m$ to $n$ occurrences. Formalized Mathematics, 15(2):53-58, 2007.

[5] Zinaida Trybulec. Properties of subsets. Formalized Mathematics, 1(1):67-71, 1990.

[6] Tetsuya Tsunetou, Grzegorz Bancerek, and Yatsuka Nakamura. Zero-based finite sequences. Formalized Mathematics, 9(4):825-829, 2001. 
[7] William M. Waite and Gerhard Goos. Compiler Construction. Springer-Verlag New York Inc., 1984.

[8] Larry Wall, Tom Christiansen, and Jon Orwant. Programming Perl, Third Edition. O'Reilly Media, 2000.

Received October 9, 2007 